\title{
Resiliencia y sentido de vida en madres de alumnos de educación especial
} Resilience and meaning of life in mothers of special education students Estela Yvana-Yupanqui ${ }^{1}$

\begin{abstract}
Resumen
Objetivo: determinar la relación entre la resiliencia y el sentido de vida en madres de alumnos de un centro educativo básico especial. Materiales y métodos: estudio de alcance correlacional y diseño de corte trans versal. La muestra estuvo conformada por 120 madres de familia de un colegio de educación especial de Los Olivos. Las variables principales fueron resiliencia y sentido de vida. Se utilizó el programa STATA 14 para el análisis de datos descriptivo e inferencial. Resultados: el $54.17 \%$ de madres presentó resiliencia moderada y un $82.5 \%$ indicadores altos de presencia de sentido de vida. Asimismo, se encontró que existe relación entre el sentido de vida y la resiliencia $(p=0.004)$, de igual forma, entre el sentido de vida con el nivel de instrucción $(p=0.015)$ y la ocupación $(p=0.026)$, respectivamente. Conclusión: el sentido de vida se relaciona con la resiliencia, ya que las situaciones difíciles orientan a las madres a replantear y cuestionar la finalidad de su realidad, ampliando la percepción del sentido de vida. Además les brinda la capacidad de poder adaptarse y hacer frente a situaciones difíciles, llegando a transformar inclusive momentos estresantes. Igualmente encontrar un sentido de vida, a pesar de las adversidades, se identificó en las madres que se dedican a las labores de la casa y que alcanzaron estudios superiores o culminó la secundaria.
\end{abstract}

Palabras clave: Resiliencia Psicológica; Educación Especial; Acontecimientos que Cambian la Vida; Estudiantes (Fuente: DeCS).

\begin{abstract}
Objective: to determine the relationship between resilience and the meaning of life in mothers of students of a special basic educational center. Materials and methods: study of correlational scope and cross-sectional design. The sample consisted of 120 mothers from a special education college in Los Olivos. The main variables were resilience and sense of life. The STATA 14 program was used for descriptive and inferential data analysis. Results: $54.17 \%$ of mothers showed moderate resilience and $82.5 \%$ had high indicators of the presence of a sense of life. Likewise, it was found that there is a relationship between the meaning of life and resilience $(\mathrm{p}=0.004)$, in the same way, between the meaning of life with the level of instruction $(\mathrm{p}=0.015)$ and occupation $(\mathrm{p}=0.026)$, respectively. Conclusion: the meaning of life is related to resilience, since difficult situations guide mothers to rethink and question the purpose of their reality, broadening the perception of the meaning of life. It also gives them the ability to adapt and deal with difficult situations, even transforming even stressful moments. Likewise, finding a sense of life, in spite of the adversities, was identified in the mothers who dedicate themselves to housework and who reached higher education or finished high school.
\end{abstract}

Keys words: Psychological Resilience; Special Education; Life Changing Events; Students (Source: DeCS).

Para citar:

Yvana E. Resiliencia y sentido de vida en madres de alumnos de educación especial. CASUS. 2018;3(3):155-160.

${ }^{1}$ CEBE Manuel Duato. Bachiller de Psicología.

Correo electrónico: estelayvana0@gmail.com
Fecha de recepción: 25-06-18

Fecha de envío a pares: 30-06-18

Fecha de aprobación por pares: 21-11-18

Fecha de aceptación: 06-12-18 


\section{INTRODUCCIÓN}

La Organización mundial de la Salud (1) señala que un $15 \%$ de la población mundial, es decir cerca de 1000 millones de personas, tiene algún tipo de discapacidad; así como grandes dificultades para su funcionamiento en general. De estas personas, 100 millones son menores de 18 años, dichas cifras indican la necesidad en los niños, adultos y sus familias de desarrollar capacidades y fortalezas para enfrentar las adversidades que se puedan presentar según la discapacidad que poseen, pues el mismo hecho de que un familiar se encuentre en una condición de discapacidad, incluye a toda la familia, modificando su equilibrio constantemente para adaptarse a las diversas circunstancias (2).

En el Perú, según el Instituto Nacional de Estadística e Informática (3), la población de niños con discapacidad ha aumentado en los últimos años, pues 10 de cada 100 personas con discapacidad son menores de edad y 1 millón 914 mil 740 están en Lima Metropolitana. Además, con ello aumentan madres que se encuentran ante una fuerte crisis existencial de tener un hijo con discapacidad y los contextos difíciles que involucra (4). En este sentido, hay evidencia que la resiliencia está ligada como soporte ante padecimientos incapacitantes (5).

La resiliencia es una característica de la personalidad que modera el efecto negativo del estrés y fomenta la adaptación (6). Se destaca la importancia de la resiliencia ante situaciones que provocan estrés y desequilibrio, viendo esta última como una capacidad de la persona, que le ayuda a sobreponerse y cambiar el enfoque (5). Asimismo, se asocia con el sentido de vida, ya que las madres de niños de educación especial tienen la capacidad de sobreponerse, enfrentarse y salir fortalecidas ante la adversidad (6). Es así que, el sentido de vida es el significado concreto de la existencia de cada individuo en un momento dado, así como la actitud que asume ante este (7).

De esta manera, la persona que está pasando por situaciones que ponen al límite sus capacidades de afrontamiento, tiene la experiencia que puede favorecer el desarrollo de la resiliencia y por ende, identificar el sentido de su vida (8).
Asimismo, la asociación entre resiliencia y sentido de vida no ha sido suficientemente estudiada en madres con hijos con discapacidad. Es por eso que esta investigación busca de algún modo aportar datos estadísticos sobre el comportamiento de estas variables. Además, indagar en la misma es de beneficio para la población seleccionada, pues a partir de los resultados se pueden plantear intervenciones más específicas para las madres y sus hijos. Por ello se planteó como objetivo determinar la relación entre la resiliencia y el sentido de vida en madres de alumnos de educación especial.

\section{MATERIALES Y MÉTODOS}

Estudio de tipo correlacional de corte transversal. La muestra estuvo conformada por 120 madres de familia de un colegio de educación básica especial de Los Olivos seleccionadas por un muestreo no probabilístico. Las variables principales fueron: resiliencia y sentido de vida.

Se define por resiliencia a aquella manera particular en que la personalidad suele adaptarse al estrés y afrontar situaciones de dificultad (6). Esta variable fue medida a través de la Escala de Resiliencia de Wagnild y Young (9), es de naturaleza categórica politómica ordinal y está categorizada en tres niveles: baja $(<120)$, moderada (121-146) y alta (>147).

El sentido de vida es el rumbo que el ser humano se fija a lo largo de la vida y que se manifiesta en una finalidad específica (10). Esta variable se midió con el Inventario de Sentido de Vida de Crumbaugh y Maholick (11), es de naturaleza categórica politómica ordinal y se clasificó en presencia de sentido de vida (>106), indefinición de sentido de vida (90-105) y ausencia de sentido de vida $(<89)$.

Las variables sociodemográficas fueron recolectadas a través de una ficha de datos sociodemográficos que consideró lo siguiente: edad de la madre, dicotómica (18-30, 31 a más); estado civil, politómica nominal (casada, conviviente, soltera, divorciada); nivel de instrucción, politómica ordinal (primaria, secundaria, técnico o universitario superior); ocupación, politómica nominal (independiente, 
ama de casa y profesional); número de hijos, dicotómica ordinal (1-2 hijos y 3 a más); edad del

Tabla 1. Descripción de las variables en madres de familia

\begin{tabular}{|c|c|c|}
\hline & $\mathbf{n}$ & $(\%)$ \\
\hline \multicolumn{3}{|l|}{ Edad } \\
\hline $18-30$ años & 29 & 24.17 \\
\hline 31 a más & 91 & 75.83 \\
\hline \multicolumn{3}{|l|}{ Estado civil } \\
\hline Casada & 43 & 35.83 \\
\hline Conviviente & 52 & 43.33 \\
\hline Divorciada & 5 & 4.17 \\
\hline Soltera & 20 & 16.67 \\
\hline \multicolumn{3}{|l|}{ Número de hijos } \\
\hline $1-2$ hijos & 82 & 68.33 \\
\hline 3 a más & 38 & 31.67 \\
\hline \multicolumn{3}{|l|}{ Nivel de instrucción } \\
\hline Primaria & 12 & 10.00 \\
\hline Secundaria & 64 & 53.33 \\
\hline $\begin{array}{l}\text { Superior técnica o } \\
\text { universitaria }\end{array}$ & 44 & 36.67 \\
\hline \multicolumn{3}{|l|}{ Ocupación } \\
\hline Profesional & 22 & 18.33 \\
\hline Ama de casa & 81 & 67.50 \\
\hline Otros & 17 & 14.17 \\
\hline \multicolumn{3}{|l|}{ Edad del hijo } \\
\hline 0 a 10 años & 106 & 88.33 \\
\hline 11 años a más & 14 & 11.67 \\
\hline \multicolumn{3}{|l|}{ Diagnóstico del hijo } \\
\hline T.E.A. & 16 & 13.33 \\
\hline Parálisis cerebral & 18 & 15.00 \\
\hline $\begin{array}{l}\text { Discapacidad } \\
\text { auditiva }\end{array}$ & 8 & 6.67 \\
\hline Síndrome de Down & 54 & 45.00 \\
\hline $\begin{array}{l}\text { Discapacidad } \\
\text { intelectual }\end{array}$ & 2 & 1.67 \\
\hline Multidiscapacidad & 10 & 8.33 \\
\hline Otros & 12 & 10.00 \\
\hline \multicolumn{3}{|l|}{ Resiliencia } \\
\hline Resiliencia baja & 15 & 12.5 \\
\hline $\begin{array}{l}\text { Resiliencia } \\
\text { moderada }\end{array}$ & 65 & 54.17 \\
\hline Resiliencia alta & 40 & 33.33 \\
\hline \multicolumn{3}{|l|}{ Sentido de vida } \\
\hline $\begin{array}{l}\text { Ausencia de sentido } \\
\text { de vida }\end{array}$ & 7 & 5.83 \\
\hline $\begin{array}{l}\text { Indefinición de } \\
\text { sentido de } \\
\text { vida }\end{array}$ & 14 & 11.67 \\
\hline $\begin{array}{l}\text { Presencia de sentido } \\
\text { de } \\
\text { vida }\end{array}$ & 99 & 82.50 \\
\hline
\end{tabular}

hijo, dicotómica ordinal (0-10 años y 11 años a más) y por último, el diagnóstico del hijo, politómica nominal (T.E.A, parálisis cerebral, discapacidad auditiva, síndrome de Down, discapacidad intelectual, Multidiscapacidad y otros)

Para el análisis estadístico se utilizó el programa STATA 14. En el análisis descriptivo se utilizaron las frecuencias y porcentajes para las variables cualitativas. A nivel inferencial, para hallar la relación entre las variables categóricas se empleó el Chi-Cuadrado con un nivel de significancia de 0.05. Esta investigación fue aprobada por el Comité de Ética de la Universidad Católica Sedes Sapientiae y la información de las participantes fue protegida de acuerdo a los principios éticos en investigación con seres humanos.

\section{RESULTADOS}

Del total de las madres involucradas en el estudio el $75.83 \%$ tuvo 31 años de edad a más. Un 43.33 $\%$ reportó el estado civil de conviviente, seguido por un $35.83 \%$ que tuvo el estado de casada. El $53.33 \%$ de ellas culminó la escuela secundaria y el $67.5 \%$ eran amas de casa. En cuanto a los hijos el $68.33 \%$ tuvo de uno a dos, el $88.33 \%$ posee una edad que oscila entre los 0 a 10 años de edad y el $45 \%$ presentó el diagnóstico de síndrome de Down. Por otro lado, se evidenció que el $54.17 \%$ de madres presentan resiliencia moderada y un $82.5 \%$ presenta indicadores altos de presencia de sentido de vida. El resto de los resultados se puede ver en la tabla 1.

Tabla 2. Asociación entre las variables principales

\begin{tabular}{lcccc}
\hline & \multicolumn{3}{c}{ Sentido de vida } & \\
& $\begin{array}{c}\text { Ausencia } \\
\text { de sentido } \\
\text { de vida } \\
\text { Indefinición } \\
\text { de sentido } \\
\text { de vida }\end{array}$ & $\begin{array}{c}\text { Presencia } \\
\text { de } \\
\text { sentido } \\
\text { de vida } \\
\mathrm{n}(\%)\end{array}$ & $\begin{array}{c}\mathbf{p} \text { - } \\
\text { value }\end{array}$ \\
\hline $\begin{array}{c}\text { Resiliencia } \\
\text { Resiliencia } \\
\text { baja }\end{array}$ & $4(26.67)$ & $2(13.33)$ & $9(60.00)$ & 0.004 \\
$\begin{array}{l}\text { Resiliencia } \\
\text { moderada }\end{array}$ & $3(4.62)$ & $6(9.23)$ & $56(86.15)$ & \\
$\begin{array}{l}\text { Resiliencia } \\
\text { alta }\end{array}$ & $0(0.00)$ & $6(15.00)$ & $34(85.00)$ & \\
\hline
\end{tabular}

Se encontró que existe relación significativa entre el sentido de vida y la resiliencia $(\mathrm{p}=0.004)$. Mostrando que el $86.15 \%$ de las madres que reportaron un sentido de vida tienen niveles moderados de resiliencia (ver tabla 2) 
Tabla 3. Asociación entre sentido de vida y las variables sociodemográficas

\begin{tabular}{lcccc}
\hline & \multicolumn{4}{c}{ Sentido de vida } \\
\cline { 2 - 5 } & $\begin{array}{c}\text { Ausencia } \\
\text { de sentido } \\
\text { de vida } \\
\mathrm{n}(\%)\end{array}$ & $\begin{array}{c}\text { Indefinición } \\
\text { de sentido } \\
\text { de vida } \\
\mathrm{n}(\%)\end{array}$ & $\begin{array}{c}\text { Presencia } \\
\text { de sentido } \\
\text { de vida } \\
\mathrm{n}(\%)\end{array}$ & $\begin{array}{c}\text { p- } \\
\text { valor }\end{array}$ \\
\hline $\begin{array}{c}\text { Nivel de instrucción } \\
\text { Primaria }\end{array}$ & $3(25.00)$ & $3(25.00)$ & $6(50.00)$ & 0.015 \\
$\begin{array}{l}\text { Secundaria } \\
\text { Superior }\end{array}$ & $2(3.13)$ & $7(10.94)$ & $55(85.94)$ & \\
$\begin{array}{l}\text { técnico o } \\
\text { universitario }\end{array}$ & $2(4.55)$ & $4(9.09)$ & $38(86.36)$ & \\
$\begin{array}{c}\text { Ocupación } \\
\text { Independiente }\end{array}$ & $0(0.00)$ & $0(0.00)$ & $17(100.00)$ & 0.026 \\
$\begin{array}{l}\text { Ama de casa } \\
\text { Profesional }\end{array}$ & $7(8.64)$ & $8(9.88)$ & $66(81.48)$ & \\
\hline
\end{tabular}

En la tabla 3, se evidenció relación entre el nivel de instrucción ( $\mathrm{p}=0.015)$, la ocupación $(\mathrm{p}=0,026)$ y el sentido de vida, respectivamente. El $86.36 \%$ de madres que reportó tener un sentido de la vida tenía un nivel de instrucción superior, seguido del $85.94 \%$ en madres que culminaron la secundaria.

Del mismo modo, un $81.48 \%$ de madres que se dedicaban a las labores de la casa registraron presencia de sentido de vida. El resto de las asociaciones no resultaron significativas.

\section{DISCUSIÓN}

Los resultados demuestran que existe una relación significativa entre el sentido de vida y la resiliencia. Además, se observó que el sentido de vida se relacionó con el nivel de instrucción y la ocupación de las madres.

Las madres que reportaron la presencia de sentido de vida poseen niveles moderados de resiliencia. Otros estudios asemejan a estos resultados señalando que hay niveles de resiliencia moderados en madres de niños de educación especial (12). Esto podría explicarse porque las madres toman una actitud positiva frente al sufrimiento, en este caso, las situaciones estresantes o dificultades que puedan presentarse al tener un hijo con discapacidad. Esta percepción del sentido de vida se ve reflejada en la misión que tienen ellas como madres a cada momento, es decir en las actividades cotidianas o las metas que se proponen con la finalidad de mantener una estabilidad en el hogar $(13,14)$.

Lo anterior puede ser posible por los diferentes mediadores externos e internos que conllevan a la adaptación y funcionamiento más óptimo de las situaciones difíciles en las que puedan estar inmersas constantemente. El hecho de tener un hijo con discapacidad crea el replanteamiento que ser madres de niños de educación especial sea visto como un proceso de aprendizaje continuo y permanente, dando así un nuevo sentido a sus vidas, volviendo a tener importancia los obstáculos que se les pueda presentar para asumirlos y enfrentarlos (5). Considerando así que no todas las situaciones difíciles se involucran de una manera negativa en las vidas de las madres participantes a la investigación; si no que es vista como una posible ganancia a nivel personal. Fomentando la adaptación y transformación a las diferentes condiciones adversas involucradas (6).

Las madres que manifestaron tener sentido de vida fueron en su mayoría las que se dedicaban a las labores domésticas. Primeramente se debe precisar que es frecuente que las madres con hijos con discapacidad renuncien a un trabajo remunerado fuera del hogar. Igualmente, que va muy de la mano la percepción del rol de ser cuidadoras de los niños y el hogar (15). Es como si fuera un compromiso de maternidad el estar constantemente para los hijos y esto las hace sentir bien (16). Demostrando así que ser madres de niños de educación básica especial fomenta que se valoren ciertos aspectos irrelevantes en la vida de los demás; todo esto como un impulso de progreso para replantear la percepción del sentido de vida y seguir adelante (5).

Los valores más altos de la presencia de sentido de vida se mostraron en las madres con un nivel de instrucción superior y de secundaria, respectivamente. Este hallazgo coincidió con otro estudio (11). Asimismo, otra investigación reportó que las madres que tienen niveles más altos de instrucción favorecen que se mejoren las oportunidades académicas para sus hijos, el nivel socioeconómico del hogar y con ello las posibilidades de que sus hijos tengan un mejor 
desempeño, a pesar de las dificultades que puedan presentar, ya que consideran a la educación como un medio para alcanzar la realización personal (17). De esta manera, se puede llegar a alcanzar el sentido de la vida, a través de una o más de las siguientes maneras: realizando una acción, teniendo algún principio o a través del sufrimiento (7).

Pues a pesar de la ocupación y el nivel de instrucción alcanzado, la madre con un niño con discapacidad tendrá esa esperanza que la ayudará a enfrentarse a las diversas dificultades, porque replanteará siempre su voluntad de sentido a la que está llamado todo hombre (7). Aquella voluntad que no depende en gran porcentaje de los recursos básicos, sino que la llevará actuar para poder transformar realidades desagradables tanto para ella como para sus hijos (6).

Se debe señalar para una mejor evaluación de los hallazgos relevantes del estudio que la población pertenece a un solo colegio, por ello se recomienda realizar investigaciones que abarquen una mayor cantidad de muestra. Así como realizar investigaciones de tipo cualitativas para una valoración más integral de los resultados.

\section{CONCLUSIONES}

El sentido de vida se relaciona con la resiliencia, ya que las situaciones difíciles orientan a las madres a replantear y cuestionar la finalidad de su realidad, ampliando la percepción del sentido de vida. Además les brinda la capacidad de poder adaptarse y hacer frente a situaciones difíciles, llegando a transformar inclusive momentos estresantes. Igualmente encontrar un sentido de vida, a pesar de las adversidades, se identificó en las madres que se dedican a las labores de la casa y que alcanzaron estudios superiores o culminaron la secundaria.

Por los hallazgos encontrados la implementación de programas centrados en la logoterapia para incrementar estrategias para hacer frente a situaciones estresantes sería de soporte para los demás integrantes de toda la familia quienes también conviven con la madre y el niño con discapacidad.

\section{REFERENCIAS BIBLIOGRÁFICAS}

1. Organización Mundial de la Salud. Informe mundial sobre la discapacidad [Internet]. Ginebra, Suiza: OMS; 2011 [citado el 04 de abril de 2018. Disponible en: http://www.who.int/disabilities/wo rld_report/2011/summary_es.pdf? ua $=1$

2. Ponce J, Torrecillas A. Estudio de factores resilientes en familiares de personas con discapacidad. International Journal of 5 . Developmental and Educational Psychology. 2014; 7(1):407-416.

3. Instituto Nacional de Estadísticas e Informática. Cerca de siete millones de niñas y niños viven en el Perú [Internet]. Lima, Perú: 6 INEI; 2016 [citado el 18 de abril de 2018]. Disponible en: https ://www.inei.gob.pe/prensa/no ticias/inei-cerca-de-7-millones-deninas-y-ninos-viven-en-el-peru9010/

Tojín A. Sentido de vida en madres que tienen un hijo diagnosticado con el trastorno del 8 . Espectro Autista. [Tesis de licenciatura]. Quetazltenango: Universidad Rafael Landívar; 2014.

Spereak R. Influencia de la resiliencia en la sobrecarga subjetiva de madres de niños con Trastorno de Espectro Autista. Revista Psicológica. 2016;6(1):25- 9 48.

Wagnild G, Young H. Development and psychometric evaluation of the Resilience Scale. Journal of Nursing Measurement.
1993;

1(2):165-178

7. Frankl V. El Hombre en busca del sentido. $12 \mathrm{a}$ ed. Barcelona: Editorial Herder; 1996.

Barbosa, R. Religiosidad, espiritualidad y comportamientos resilientes. IV Congreso Internacional de Investigación y Práctica Profesional en Psicología XIX Jornadas de Investigación VIII Encuentro de Investigadores en Psicología del MERCOSUR. 2012.

Novella A. Incremento de la resiliencia luego de la aplicación de un programa de psicoterapia breve en madres adolescentes [Tesis de maestría]. Lima: Universidad Mayor de San Marcos; 2017. 
10. Ramírez C, Saavedra C. El sentido de la vida [Tesis de maestría]. Antioquía: Universidad de Antioquía;

1981. 13. García J. La logoterapia de Viktor

11. Bartschi C. Evaluación del sentido de vida y la búsqueda de metas noéticas desde el punto de vista de la logoterapia en estudiante universitarios [Tesis de licenciatura]. Lima: Universidad de Lima; 1999

12. Briones $\mathrm{P}$, Tumbaco C. Resiliencia materna y ajuste parental ante la discapacidad en madres de Centros Educativos Básicos Especiales de Lima Este, 2016 [Tesis de Frankl. Revista de espiritualidad. 2005;64(1):279-312.

licenciatura]. Lima: Universidad Peruana Unión; 2017

14. Gómez M. El sentido de vida de las madres de niños prematuros internados en Unidad de Cuidados Intensivos Neonatales (UCIN). Revista de Psicología Ciencias Afines: Interdis ciplinaria. 2016;33(1):5-19.

15. Aguilar D, Medina B, Martínez R. El sentido de vida en madres solas. Revista científica y profesional de la Asociación Latinoamericana para la Formación y la Enseñanza de la Psicología. 2017;5(13):121131.

16. Grupo de Investigación AFIN Experiencias de madres con hijos con discapacidad [Internet]. Barcelona, España: AFIN; 2015. Disponible en: https://ddd.uab.cat/pub/afin/afinSP A/afin_a2015mln68iSPA.pdf.

17. Arancibia V. El rol de la madre como mediadora en el proceso de habilitación. Revista de Estudios Públicos. 1995; 59:251-264. 